\title{
Somatic gene therapy for genetic disease
}

Recent advances in recombinant DNA technology have led to an increase in our understanding of the molecular basis of many genetic diseases. Approximately 3500 different human genetic diseases are known, and as the genes responsible for these diseases are identified and cloned, many advances in treatment and maintenance will be made. These advances have already translated into improved methods for the prenatal diagnosis of many diseases and the use of recombinant gene products in treatment regimes. It is envisaged, however, that with further developments it will soon be possible to correct the genetic defects themselves in affected individuals, through the use of somatic gene therapy techniques. The potential for this form of treatment has generated much interest and the possible applications of somatic gene therapy have been much discussed. ${ }^{1-4}$ For the purposes of this annotation, however, we are confining the discussion to those genetic diseases which are currently treated by bone marrow transplantation.

Somatic gene therapy is defined as the correction of a defective or absent gene with its cloned functional equivalent. It differs from germline gene therapy in that the gene is only introduced into the somatic cells of the patient and not into the germline (that is, sperm or ova). Therefore, the gene can only be expressed in those cells into which it was introduced and their progeny cells, but it cannot be passed on to subsequent generations of children.

Most cases of genetic disease are the result of a point mutation rather than a gene deletion, and this means that the defective gene is still present, though not necessarily active, in the affected cells. Therefore, the introduction of a functional gene in addition to the defective gene would result in gene augmentation. However, because of the nonspecific nature of the integration event where the gene is inserted randomly into the human genome, a variety of problems may arise. These could include non-expression of the introduced gene or insertional mutagenesis, whereby a normally active gene is silenced or a silent deleterious gene may become activated. Despite these potential problems, this model has been the most widely studied so far and currently offers the best prospect for the treatment of genetic diseases.

A much more difficult technique would be true gene replacement whereby the defective gene is removed from the affected cells and replaced with a functional gene in the correct orientation and chromosomal location. Such gene replacement would be technically difficult, although a model for gene targeting involving homologous recombination is being developed in the mouse ${ }^{5}$; this is currently only applicable to germline gene therapy.

\section{Methods of gene insertion}

A variety of methods for the introduction of cloned genes into cells have been suggested and these can be classed into two groups: physical and viral. The physical methods include coprecipitation with calcium phosphate and the use of polycations or lipids to complex with DNA, cell fusion techniques using liposomes, erythrocyte ghosts and protoplasts, microinjection, and electroporation techniques. While large numbers of cells can be treated at once (except by microinjection) these techniques have the disadvantage that the efficiency of integration is low and multiple copies of the DNA are usually inserted.

The first viruses used in gene expression experiments were the DNA tumour viruses (SV40, polyoma) and the adenoviruses. Recently the work has focused on murine retroviral vectors, particularly those based on Moloney murine leukaemia virus.

Retroviruses have a number of useful features that makes them suitable for use as vectors for gene therapy. In contrast with the physical methods of introducing DNA into cells, retroviral vectors can infect up to $100 \%$ of target cells and the DNA is usually integrated as a single copy. Once integrated into the host genome the provirus acts as a cellular gene in that it is faithfully passed onto progeny cells. The production of progeny virus is non-lytic, leading to the establishment of permanent, viral producing cell lines which give high titre virus for infection. ${ }^{6}$ The proteins required for viral encapsidation (the gag, pol and env genes) can be supplied in trans, which means that replication defective retroviruses can be produced that are capable of only one round of infection, which removes the problem of constant reinfection of cells. The possibility exists, however, that infective viruses could be produced subsequent to gene insertion if they recombine with endogenous retroviruses. Furthermore, retroviral vectors can only encode up to seven kilobases of introduced DNA, which in most cases is not sufficient to encode a full length gene and its appropriate control sequences. Loss of control sequences may lead to inappropriate tissue expression and low levels of expression of the gene product.

In the future it is possible that more efficient, higher titre viral vectors capable of encoding larger pieces of DNA may be developed based on vaccinia, adenoassociated, herpes, bovine papilloma, and other viruses.

\section{Criteria for somatic gene therapy}

There are a large number of genetic diseases that could potentially be treated by somatic gene therapy. The candidate disorders for this type of treatment must meet the following criteria: (i) the disorder must have a severe and predictable phenotype, (ii) the defective gene must have been identified and cloned, (iii) expression of the gene product should not require precise regulation, nor particularly high levels of expression to correct the defect, and (iv) there must be a suitable delivery system for the implantation of the genetically modified cells.

Currently, the most suitable disorders for this type of treatment are diseases that affect the hematopoietic system. The main reason for this is that there already exists a suitable method for the reintroduction of the treated cells through bone marrow transplantation. Indeed, diseases such as severe combined immunodeficiency due to adenosine deaminase deficiency may be successfully treated by allogeneic bone marrow transplantation. ${ }^{7}$ The gene for adenosine deaminase has been cloned and a considerable amount of effort has already been put into developing strategies for the treatment of this disease by somatic cell gene therapy, ${ }^{2}$ although enzyme replacement treatment with polyethylene glycol stabilised adenosine deaminase presents another possibility. ${ }^{8}$ Other candidate disorders of this type currently include purine nucleoside phosphorylase deficiency, chronic granulomatous disease, Gaucher's disease, and the haemoglobinopathies such as the thalassaemias and sickle cell disease.

The idea of introducing genes into bone marrow stem cells is particularly attractive as these cells should 
permanently correct the genetic defect. Indeed, studies in mice have shown that genes can be stably introduced into bone marrow stem cells by retroviruses and expression in differentiated progeny cells has been detected for some time afterwards. $^{9-11}$ Although stem cells have been identified with some certainty in mice, ${ }^{12} 13$ there is still some way to go in defining their human equivalents. ${ }^{14} 15$ There also remains the problem of putting the human stem cells into cycle, as proviral integration is dependent on DNA replication and at present there is no efficient means of achieving this. Another problem is the question of gene regulation. In order to get tissue specific gene expression at the appropriate level it is necessary for the gene to be under the control of appropriate regulatory elements and in the vast majority of cases, with few notable exceptions, ${ }^{16} 17$ these elements have yet to be defined.

\section{Ethical considerations}

Apart from the technical problems of ensuring tissue specificity and adequate levels of gene expression there remain a number of medical and ethical issues. First, for diseases in which the gene product is produced by bone marrow derived cells, in some cases, it may be necessary to create space within the bone marrow compartment in order to decrease competition between infected and uninfected cells thereby providing a selective advantage for the stem cells carrying the functional gene. The currently used chemotherapy or radiotherapy techniques for bone marrow ablation may be sufficient but eventually monoclonal antibodies targeted at the relevant cells in the bone marrow may provide a less toxic alternative. Complete marrow ablation may not be necessary as it has been shown in the partially ablated thalassaemic mouse model that the introduction of only $5 \%$ of normal bone marrow cells may correct the defect. ${ }^{18}$

It will probably be necessary to define the nature of the defect in individual cases as in the small number of families where a gene deletion rather than a point mutation exists the product of the introduced gene may prove to be immunogenic, thereby rendering the treatment ineffective. The techniques currently being developed allow the potential for insertional oncogenesis, as they all rely on random integration events. The only foreseeable way to overcome this problem is by gene targeting but this remains only a theoretical possibility at present.

In view of these problems, somatic gene therapy should, at present, only be considered for diseases that are fatal in early infancy or that carry a very poor prognosis with the currently available treatments. There needs to be an ethical debate in the United Kingdom to discuss issues well before the first attempt at treatment are contemplated. In the United States the machinery to obtain ethical approval is already in place whereby permission needs to be obtained from national bodies. No such national body exists in the
United Kingdom and there is the potential risk that such treatments will be carried out under the approval only of individual hospital ethical committees, the performance of which may vary widely. The European Medical Research Councils made a joint statement over a year ago to the effect that a national body in each country should consider all proposals for human gene therapy and ensure the application of agreed national guidelines. They recommended that early trials should be monitored by a central body. ${ }^{19}$ As most of the diseases that are currently being contemplated as suitable for such treatment affect the paediatric age group it may be appropriate for the British Paediatric Association to take part in the formulation of suitable ethical policies and in the formation of such a national body to assess and monitor the trials.

C KINNON

Department of Immunology, R J LEVINSKY Institute of Child Health, 30 Guilford Street London WCIN IEH

1 Anderson FW. Prospects for human gene therapy. Science 1984;226:401-9. 2 Kantoff PW, Freeman SM, French Anderson W. Prospects for gene therapy for immunodeficiency diseases. Annu Rev Immumol 1988;6:581-94.

3 Williams DA. Gene transfer and the prospects for somatic gene therapy. Hematology and Oncology Clinics of North America 1988;2:277-87.

4 Friedmann T. Progress toward human gene therapy. Science 1989;244 1275-81.

5 Capecchi MR. Altering the genome by homologous recombination. Science 1989;244:1288-92.

6 Cone RG, Mulligan RC. High-efficiency gene transfer into mammalian cells generation of helper-free combinant retrovirus with broad mammalian host generation of helper-free combinant retrovirus with

7 Levinsky RJ. Recent advances in bone marrow transplantation. Clin Immunol Immunopathol 1989;50:124-32.

8 Hershfield MS, Buckley RH, Greenberg ML, et al. Treatment of adenosine deaminase deficiency with polyethylene glycol-modified adenosine deaminase. $N$ Engl f Med 1987; 316:589-95.

9 Dick JE, Magli MC, Huszar D, Phillips RA, Bernstein A. Introduction of a selectable gene into primitive stem cells capable of long-term reconstitution of the hemopoietic system of W/Wv mice. Cell 1985;42:71-9.

10 Keller G, Paige C, Gilboa E, Wagner ER. Expression of a foreign gene in myeloid and lymphoid cells derived from multipotent haematopoietic precursors. Nature 1985;318: 149-54.

11 Dzierzak EA, Papayannopoulou T, Mulligan RC. Lineage-specific expression of a human $b$-globin gene in murine bone marrow transplant recipients reconstituted with retrovirus-transduced stem cells. Nature 1988;331: reconstitu 41 .

12 Visser JWM, Bauman JCJ, Mulder AH, Eliason JF, deLeeun AM. Isolation of murine pluripotent hemopoietic stem cells. F Exp Med 1984;159. $1576-90$.

13 Spangrude GJ, Heimfeld S, Weissman IL. Purification and characterization of mouse hematopoietic stem cells. Science 1988;241:58-61.

14 Watt SM, Karhi K, Gatter K, et al. Distribution and epitope analysis of the cell membrane glycoprotein (HPCA-1) associatd with human hemopoietic cell membrane glycoprotein (HPCA-1) associa

15 Berenson RJ, Andrews RG, Bensinger WI, et al. Antigen CD34+ marrow cells engraft lethally irradiated baboons. F Clin Invest 1988;81:951-5.

16 Greaves DR, Wilson FD, Lang G, Kioussis D. Human CD2 3'-flanking sequences confer high-level, $\mathrm{T}$ cell-specific, position-independent gene expression in transgenic mice. Cell 1989;56:979-86.

17 Talbot D, Collis P, Antoniou M, Vidal M, Grosveld F, Greaves DR. A dominant control region from the human $\mathrm{b}$-globin locus conferring integration site-independent gene expression. Nature 1989;338:352-5.

18 Wagemaker G, Visser TP, van Bekkum DW. Cure of murine thalassemia by bone marrow transplantation without eradication of endogenous stem cells. Transplantation 1986;42: 248-51.

19 European Medical Research Councils. Gene therapy in man. Recommenda tions of European Medical Research Councils. Lancet 1988;i:1271-2. 